\title{
Long-term biofouling formation mediated by extracellular proteins in Nannochloropsis gaditana microalga cultures at different medium $\mathrm{N} / \mathrm{P}$ ratios
}

\author{
Y. Soriano-Jerez ${ }^{1}$, Lorenzo López-Rosales ${ }^{2}, \mathrm{M}^{\mathrm{a}}$ Carmen Cerón-García ${ }^{1}$, Asterio \\ Sanchez-Miron $^{3}$, Juan Gallardo Rodríguez ${ }^{1}$, Francisco García ${ }^{1}$, and Emilio Grima ${ }^{2}$ \\ ${ }^{1}$ University of Almería \\ ${ }^{2}$ Universidad de Almeria \\ ${ }^{3} \mathrm{UAL}$
}

August 28, 2020

\begin{abstract}
Biofouling represents an important limitation in photobioreactor cultures. The biofouling propensity of different materials (polystyrene, borosilicate glass, polymethyl methacrylate and polyethylene terephthalate glycol-modified) and coatings (two spray-applied and nanoparticle-based superhydrophobic coatings and a hydrogel-based fouling release coating) was evaluated by means of a short-term protein test, using bovine serum albumin (BSA) as a model protein, and by the long-term culture of the marine microalga Nannochloropsis gaditana under practical conditions. The results from both methods were similar, confirming that the BSA test predicts microalgal biofouling on surfaces exposed to microalgae cultures; these secrete macromolecules, such as proteins, that have a high capacity for forming a conditioning film prior to cell adhesion. The hydrogel-based coating showed significantly reduced BSA and N. gaditana adhesion, whereas the other surfaces failed to control biofouling. Microalgal biofouling was associated with an increased concentration of sticky extracellular proteins at low N/P ratios (below 15).
\end{abstract}

\begin{abstract}
Biofouling represents an important limitation in photobioreactor cultures. The biofouling propensity of different materials (polystyrene, borosilicate glass, polymethyl methacrylate and polyethylene terephthalate glycol-modified) and coatings (two spray-applied and nanoparticle-based superhydrophobic coatings and a hydrogel-based fouling release coating) was evaluated by means of a short-term protein test, using bovine serum albumin (BSA) as a model protein, and by the long-term culture of the marine microalga Nannochloropsis gaditana under practical conditions. The results from both methods were similar, confirming that the BSA test predicts microalgal biofouling on surfaces exposed to microalgae cultures; these secrete macromolecules, such as proteins, that have a high capacity for forming a conditioning film prior to cell adhesion. The hydrogel-based coating showed significantly reduced BSA and N. gaditana adhesion, whereas the other surfaces failed to control biofouling. Microalgal biofouling was associated with an increased concentration of sticky extracellular proteins at low N/P ratios (below 15).
\end{abstract}

Keywords: microalgae, cell adhesion, extracellular proteins, N/P ratio, extracellular organic substances

\section{Introduction}

Biofouling represents a major limitation for the long-term culture of microalgae in photobioreactors (Zeriouh et al., 2019a; Zeriouh et al., 2017b). The ubiquity of cells and extracellular organic substances (EOS) secreted by microalgae in industrial photobioreactors (PBRs) creates a competition in both to colonize the PBR 
walls in contact with the culture. Since the adhesion process timescale for EOS, such as proteins, is shorter (seconds to hours) than that for cell adhesion (hours to days), the initial conditioning film covering the PBR walls mainly consists of EOS. Nonetheless, this conditioning film accelerates cell adhesion on surfaces. The biofouling that forms is extremely difficult to prevent or eliminate. The use of in-situanti-biofouling approaches, such as increasing the turbulence in the culture, generally leads to cells that are more resistant to detachment being selected. This results in the development of very sticky biofilms.

A wide variety of anti-biofouling materials and coatings considered for use in PBRs has been studied (Talluri et al., 2020; Wang et al., 2017; Wang et al., 2020; Zeriouh et al., 2019a). All of them have failed to completely prevent long-term biofouling. They were selected based on how their surface properties might interact with the cells' surface properties, not with those of the EOS. Even though proteins and proteinaceous materials are thought to be the most abundant class of EOS components in marine microalgae cultures, protein adsorption has hardly been considered as the origin of cell adhesion. Therefore, anti-biofouling studies looking at microalgae-based bioprocesses should evaluate at least two aspects: (i) the propensity of surfaces to protein adherence; and (ii) the effect of the culture conditions on EOS production. The quantity, composition, structure, and properties of the EOS produced are influenced by a wide variety of abiotic factors (Xiao and Zheng, 2016). However, the nitrogen to phosphorous ratio (N/P) in the microalgae culture medium exerts a remarkable modulating effect on EOS production (Xiao and Zheng, 2016).

Small-scale culture devices in orbital shakers are commonly used to study microorganism biofilms and to assess the effect exerted by shear stress on cell proliferation and attachment. Nonetheless, they present a complex three-dimensional flow pattern, varying in both time and space. Understanding flow topology has been related to the shear stress distribution in these culture devices, which has helped explain the cell biofilm distributions observed (Salek et al., 2011). CFD simulations have also been used to resolve the flow structures in these devices (Berson et al., 2008).

This work aims to evaluate the biofouling susceptibility of different materials and coatings immersed in cultures of the marine microalgaNannochloropsis gaditana, a species used as model in studies on biofouling formation in photobioreactors (Zeriouh et al., 2019a; Zeriouh et al., 2019b; Zeriouh et al., 2017a). As a first step, the surfaces were tested in short-term batch adhesion experiments with the model protein BSA (Wang et al., 2017). In the second step, microalgal adhesion experiments on long-term cultures were carried out in an orbitally agitated transparent laboratory vessel acting as a simulator of real flow conditions. The long-term cultures were performed at different initial N/P ratios in the culture medium to alter the cells' EOS production. The results allowed us to assess the suitability of the short-term BSA adhesion test as a predictor of biofouling and the effectiveness of selected surfaces for biofouling control.

\section{Materials and Methods}

\subsection{Preparation of materials and coated coupons}

The rigid materials used in the test were polystyrene (PS), borosilicate glass (GS), polymethyl methacrylate (PMMA) and polyethylene terephthalate glycol-modified (PETG); PS was opaque and the remaining materials were transparent. All were cut in the form of rectangular coupons $(1.5 \mathrm{~cm} \mathrm{x} 2.5 \mathrm{~cm} \mathrm{x} 0.1 \mathrm{~cm}$ and $2 \mathrm{~cm} \mathrm{x}$ $2.5 \mathrm{~cm} \times 0.1 \mathrm{~cm}$ ). Since PMMA is a widely used material in the manufacture of PBRs, PMMA coupons were utilized to prepare the following commercially available coatings: (a) a translucent, spray-applied superhydrophobic coating (NeverWetß, NeverWet LLC, USA; NW); (b) a transparent hydrophobic coating with

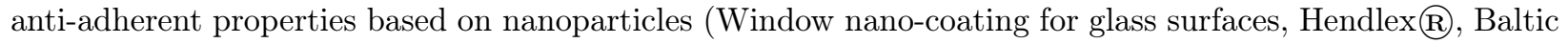
Nano Technologies, Lithuania; HX); and (c) an opaque non-toxic hydrophobic fouling release coating (FRC) that provides an invisible antibiofouling hydrogel microlayer (Hempasil $\mathrm{X}_{3} \mathrm{R}$, Hempel A/S, Denmark; H-X3). The preparation of the three coatings was carried out by strictly following the manufacturers' instructions. The H-X3 and HX coatings were applied with a wet film thickness of $150 \mu \mathrm{m}$ using a micrometer adjustable film applicator (3580/3 Universal micrometer applicator, Neurtek, Spain).

The GS coupons was cleaned as described by Ozkan and Berbeglu (2013a). The PMMA, PS and PETG were cleaned as detailed elsewhere (Ruiz-Cabello et al., 2011), except for using acetone on the PS due to 
chemical incompatibility. The coatings were washed with abundant deionized and sterilized water.

The media surface roughness $\left(R_{a}\right.$; the arithmetic media deviation from the mean line within the assessment length) was measured with a surface profiler (PCE-RT 11, PCE Ibérica S.L., Albacete, Spain) with a 1mm scan length and a $0.111 \mu \mathrm{m} /$ sample resolution. Table 1 describes the $R_{a}$ data for all the surfaces assayed.

\subsection{Physicochemical properties of the materials and coatings used}

The contact angles on the different surfaces described above were measured by means of the sessile drop method, using a goniometer (Drop Shape Analyzer DSA25, KRÜSS GmbH, Germany). Two polar liquids (water and formamide) and another apolar liquid (diiodomethane) were used as reference liquids. As formamide was chemically incompatible with the HX and NX coatings, it was replaced by glycerol. However, NW was not only incompatible with diiodomethane, but also with a wide variety of apolar liquids commonly used in contact angle measurements (bromonaphthalene, hexadecane, dodecane, decane, ethanol, chloroform and toluene). Consequently, the contact angle for the apolar liquid was not determined for NW. All measurements were performed on duplicate coupons. Before measuring the contact angles, the coupons were rinsed following the washing protocol with abundant deionized water, and then allowed to dry at room temperature.

From the contact angles measured, the different surface energy components $\left(\gamma_{s}^{\mathrm{LW}}, \gamma_{s}^{+}, \gamma_{s}^{-}, \gamma_{s}^{\mathrm{AB}}\right)$ were calculated, as was the change in the free energy of cohesion $\left(\Delta G_{\text {iwi }}\right)$, the water adhesion tension with the surface $\left(\tau^{\circ}\right)$ and the critical surface tension $\left(\gamma_{S},\right)$ for each surface, as described earlier (Zeriouh et al., 2019a). Table 1 shows the values of the above parameters.

The surfaces used vary greatly in terms of hydrophobicity, from hydrophobic surfaces $\left(\vartheta \mathrm{w}>65^{\circ}\right)$, almost even superhydrophobic $\left(\vartheta \mathrm{w}>150^{\circ}\right)$ in the case of the NW coating, to hydrophilic surfaces $\left(\vartheta \mathrm{w}<65^{\circ}\right)$, such as glass. Taking the $\tau^{0}$ parameter into account, one can distinguish the purely hydrophobic surfaces as being HX, PS, NW and H-X3. Nevertheless, all the surfaces can be considered smooth (with $R_{a}$ values ranging from $0.02-0.06 \mu \mathrm{m}$ ) except for the NW coating, which had an $R_{a}$ of $5 \mu \mathrm{m}$. A surface is considered smooth when it has $R_{a}$ values below $0.6 \mu \mathrm{m}$. The only non-smooth surface was NW due to the texture effect intentionally introduced by the manufacturer, in an attempt to obtain the lotus effect .

\subsection{Short-term BSA batch adhesion test}

A model protein, bovine serum albumin (BSA), was used to evaluate the propensity of the different materials and coatings to protein adherence. The BSA adhesion test was carried out as described elsewhere (Wang et al., 2017). Briefly, the coupons were immersed in a BSA solution prepared in PBS at a concentration of $1 \mathrm{mg}$ $\mathrm{ml}^{-1}(\mathrm{pH} 7.2-7.4)$ and kept at $25^{\circ} \mathrm{C}$ for $4 \mathrm{~h}$ with orbital shaking at $100 \mathrm{rpm}$. Transparent $15 \mathrm{ml}$ polypropylene Petri plates, with a 50-mm internal diameter, were used as incubation vessels. Next, the samples were rinsed with fresh PBS and transferred to clean Petri plates where they were washed in an aqueous solution of 1 wt\% sodium dodecyl sulfate (SDS). After being shaken for $20 \mathrm{~min}$ and sonicated for $10 \mathrm{~min}$, the protein concentration in the SDS solution was determined by spectrophotometric bicinchoninic acid (BCA) assay, measuring the absorbance at $562 \mathrm{~nm}$. The amount of protein adsorbed on the surface was expressed as $\mu \mathrm{g}$ BSA mm $\mathrm{m}^{-2}$. The measurements were performed on duplicate coupons for each surface.

\subsection{Long-term biofouling experiments}

\subsubsection{Microalgae, culture conditions and experimental setup}

The marine microalga Nannochloropsis gaditana B-3 was used. It was provided by the Marine Culture Collection at the Andalusian Institute of Marine Sciences (CSIC, Cádiz, Spain). Inoculum was grown in $1 \mathrm{~L}$ spherical flasks under a continuous $(12: 12 \mathrm{~h})$ light-dark illumination regimen at $25 \pm 2{ }^{\circ} \mathrm{C}$, the illumination being provided by $32 \mathrm{~W}$ fluorescent lamps rendering an average irradiance of $100 \mu \mathrm{E} \mathrm{m}^{-2} \mathrm{~s}^{-1}$ on the culture flask surface. The flasks were agitated by filter-sterilized air sparging injected through a sparger nozzle at an aeration rate of $0.5 \mathrm{vv}^{-1} \mathrm{~min}^{-1}$. The culture medium was prepared from natural, filter sterilized $(0.22$ $\mu \mathrm{m}$ Whatman GF/F $47 \mathrm{~mm}$, Maidstone, The United Kingdom) Mediterranean seawater with 30 psu. The 
preparation of the culture medium (N-optimized ALGAL medium) and its exact composition is described elsewhere (Camacho-Rodríguez et al., 2013).

Transparent $750 \mathrm{~mL}$ polypropylene conical-frustum vessels, with an interior diameter of $62 \mathrm{~mm}$, an exterior diameter of $94 \mathrm{~mm}$ and a height of $141 \mathrm{~mm}$, were used as culture systems in the experiments. The seven different materials and coatings were used after being prepared as indicated above. The coupons with the $1.5 \mathrm{~cm} \times 2.5 \mathrm{~cm} \times 0.1 \mathrm{~cm}$ dimensions were placed on the wall of each vessel, whereas those of $2 \mathrm{~cm} \mathrm{x} 2.5 \mathrm{~cm}$ $\mathrm{x} 0.1 \mathrm{~cm}$ were placed on the bottom. Thus, each vessel had three replicates of each material and coating, fourteen coupons on the wall and seven on the bottom. All the coupons were fixed to the vessels with the help of a harmless hot melt adhesive (Parkside PNKPZ 3 B2, Lidl, Neckarsulm, Germany).

All the vessels were sterilized by rinsing with sodium hypochlorite solution and subsequent neutralization with sodium thiosulfate (Andersen, 2005). The vessels were then held on an orbital shaker with a $3 \mathrm{~cm}-$ diameter shaking orbital (OVAN MAXI, OL30-ME, Barcelona, Spain) and maintained at $25 \pm 2{ }^{\circ} \mathrm{C}$ in a thermostatic chamber. They were illuminated with cool daylight lamps (Phillips PL-32 W/840/4p) under a 12:12-h light/dark cycle. The average irradiance at the surface of the vessels was $110 \mu \mathrm{mol} \cdot \mathrm{m}^{-2} *_{\mathrm{s}}{ }^{-1}$. The irradiance was measured using a $4 \pi$ sensor (QSL-2101; Biospherical Instruments, San Diego, CA, USA). The vessels were shaken continuously at $120 \mathrm{rpm}$.

The N-optimized ALGAL medium was used as a basis for assaying the different molar N/P ratios $(5,15,45$, 60 and 90 ) by changing the phosphorous $(\mathrm{P})$ concentration and fixing the nitrate concentration $(11.3 \mathrm{mM})$. The experiments were inoculated with exponentially growing cells and acclimated to the irradiance level of the experiments at an initial biomass concentration of $0.12 \pm 0.01 \mathrm{~g} \cdot \mathrm{L}^{-1}$ and an initial $\mathrm{pH}$ of 7.8 . The working volume was $200 \mathrm{~mL}$. Two culture modes were assayed in this temporal sequence: batch and fed-batch mode with a pulse-feeding strategy. Fed-batch mode started once the stationary growth phase was reached in batch mode. In fed-batch mode, concentrated medium stocks were added every time a stationary growth phase was reached. For this, $5 \mathrm{~mL}$ of culture was replaced by an equal volume containing a nutrient stock equivalent to $200 \mathrm{~mL}$ of the medium used. The stationary state was assumed to have been achieved when no significant changes $(+-10 \%)$ in biomass concentration were recorded over three consecutive days. The culture samples were collected, centrifuged at $7000 \mathrm{~g}$ for $5 \mathrm{~min}$, washed with a $0.5 \mathrm{M}$ ammonium bicarbonate solution (Zhu et al., 2013) and then freeze-dried. The dry biomass and supernatants were immediately analyzed or stored frozen at $-22 \operatorname{deg}$. The culture experiments were performed in duplicate.

\subsubsection{Monitoring microalgal growth and attachment}

The biomass concentration in the culture vessels $\left(C_{b}, \mathrm{~g}\right.$ d.w. $\left.\mathrm{L}^{-1}\right)$ was estimated by applying a calibration curve between $C_{b}$ and $O D_{540}$, as published previously (Camacho-Rodriguez et al., 2013):

$$
\overline{C_{b}=0.257 \bullet \mathrm{OD}_{540} ; \quad r^{2}=0.9916 \quad[1]}
$$

where $O D_{540}$ is the absorbance at $540 \mathrm{~nm}$ (measured using a Helios Omega UV-Vis spectrophotometer, Thermo Scientific, Horsham, England). The photosynthetic efficiency of Photosystem II $\left(F_{v} / F_{m}\right)$ for the freely-suspended cells in the culture broth was recorded with a pulse amplitude modulation (PAM) chlorophyll fluorometer (PAM-2500 chlorophyll fluorometer, Heinz Walz GmbH, Effeltrich, Germany), as described elsewhere (López-Rosales et al., 2014). $F_{v} / F_{m}$ is unanimously considered to be an indicator of cell stress. All determinations were performed in triplicate and the average value was used.

Regarding the attachment of the cells to the coupons, once the cultivation experiments were finished, the coupons were carefully detached from the vessels and transferred into a flask filled with plenty of sterile Mediterranean seawater to remove non-adherent cells and debris. The microalgae adhesion intensity on the coupons was evaluated using a procedure based on the Chla fluorescence of the attached algae cells, as previously described (Zeriouh et al., 2017a). 


\subsubsection{Supernatant characterization}

The phosphate and nitrate in the supernatants were measured using the 4500-P and 4500-N spectrophotometric methods for the examination of water, as published by the American Public Health Association (APHA, 1995). The protein concentration in the supernatants was quantified using a bicinchoninic acid protein assay kit (Catalog № BCA1 and B9643, Merck KGaA, Darmstadt, Germany). The ammonium-nitrogen $(\mathrm{NH} 4+-\mathrm{N})$ in the supernatants was measured calorimetrically using Nessler's method (protocol D1426-08, as proposed by the American Society for Testing and Materials (D1426-08, 2008)). The total concentration of amino acids was quantified as described elsewhere (Nielsen et al., 2001). All determinations were performed in triplicate and the average value was used.

\subsubsection{Other analytical measurements.}

The total phosphorous and nitrogen contained in the biomass and supernatants were determined as phosphate (4500-P) and nitrate (4500-N) after applying a modified chemical wet-oxidation method, as previously reported (Molina-Miras et al., 2018). The protein content in the biomass was measured, as described by González-López et al. (2010). All determinations were performed in triplicate and the average value was used.

\subsubsection{Toxicity assessment of the different materials and coatings}

To evaluate the potential toxicity of the different types of coupons assayed in the study, 21 coupons of the same rigid material or coating were affixed to the same transparent polypropylene conical-frustum vessel (14 on the side wall and 7 on the bottom), as described in Section 2.4.1. The control vessel consisted of a vessel that did not contain coupons.

$N$. gaditana was grown in batch mode in all the vessels for 15 days. The culture conditions and procedure were the same as those detailed in Section 2.4.1. The toxicity was assessed at the beginning and at the end of the culture.

Coupon toxicity was evaluated using the two methods described earlier (Zeriouh et al., 2017a): (i) the maximum photochemical photosystem II (FV/FM) yield; and (ii) the rapid light curve (RLC) technique for measuring the photosynthesis-light response curves (White \& Critchley, 1999). After dark-cell acclimation for 30 minutes, the Fv/Fm was measured, and subsequently the RLCs were obtained with a 27 s exposure at each actinic light level. The photosynthetic activity estimated as the relative electron transport rate (rETR) at incremental irradiances, $E\left(0,10,60,140,242,411,661,997,1381\right.$, and $1963 \mu \mathrm{mol}$ photons $\mathrm{m}^{-2}$ $\mathrm{s}^{-1}$ ), allowed us to obtain a complete RLC, fitting the rETR vs $E$ data to the Eilers and Peeters' model (Eilers and Peeters, 1988). The photosynthetic parameters calculated were $a$ (the photosynthetic rate in the light-limited region), $E_{k}$ (the saturating irradiance) and $r E T R_{\max }$ (the relative maximum electron transport rate). All experiments were carried out in duplicate vessels, with duplicate sampling in each vessel, and the average value was used. The values (data not shown) of the above parameters did not vary significantly compared to the controls throughout the cultivation period, indicating that these materials and coatings are not toxic toN. gaditana, even after long-term exposure.

\subsection{Fluid-dynamic characterization in the culture vessels}

Computational fluid dynamics were used to simulate and characterize the flow field, and to map the velocity and strain-rate fields.

The time-dependent simulations were performed using ANSYS Fluent@ $\mathbb{R}$ v2020R1 (www.ansys.com) software. As we were interested in the velocity and strain rate values close to the coupons, the SST k- $\omega$ turbulence model was used. An implicit two-phase VOF model was employed to track and locate the liquid free surface. The liquid velocity at the solid walls was taken to be zero (i.e. non-slip conditions). In all the simulations, the liquid was seawater (density $=1025 \mathrm{~kg} \cdot \mathrm{m}^{-3}$, viscosity $=1.012 \times 10^{-3} \mathrm{~Pa} \mathrm{~s}$ ) and the air was in the gas phase (density $=1.225 \mathrm{~kg}^{*} \mathrm{~m}^{-3}$, viscosity $=1.789 \times 10^{-5} \mathrm{~Pa} \mathrm{~s}$ ). The viscosity of the cell suspension at the inoculation time was the same as seawater. The viscosity was checked throughout the culture period and no 
measurable variations in the culture viscosity were observed over this time. Therefore, seawater properties were maintained in all the simulations. A water-air surface tension of $72.5 \times 10^{-3} \mathrm{~N}^{*} \mathrm{~m}^{-1}$ was used.

The simulations utilized a pressure-based model under transient conditions. Gravity was included in the model in the negative z-direction. The pressure reference was fixed at the mouth of the flask. The other models employed were SIMPLEC for the pressure-velocity coupling, the Least Square Cell-Based for Gradient scheme for spatial discretization, QUICK for momentum, compressive for volume fraction, and second-order upwind discretization for turbulent kinetic energy and the dissipation rate.

The time-step size was fixed at $1 \times 10^{-3} \mathrm{~s}$ with a maximum of 25 iterations per time step to ensure a maximum CFL value of 1 , and that the residuals dropped four orders of magnitude. The flow stabilized after 9 seconds.

The optimum mesh contained around 1.6 million computational grids, created using the tetra mesh option of ANSYS(r). 15 inflation layers were created on the surface of every coupon. The first cell had a size of 5 $\mu \mathrm{m}$. The mesh was converted to polyhedral in Fluent to ensure that the cells were aligned with the rotating flow to minimize the numerical error diffusion.

The flask movement was simulated as a moving mesh. The clockwise orbit of the flask

was specified in a user-defined function (UDF) in terms of the orbital angular velocity and orbital radius.

\subsection{Statistical analyses}

A one-way ANOVA test was used for significant difference analysis. Multifactor ANOVA were performed to determine the effect of the following factors and their interactions on long-term biofouling results: N/P (factor A), coupon (i.e. type of material and coatings; factor B), coupon position in the culture vessels (wall or bottom; factor C) and interactions (A-B; A-C; B-C and A-B-C). Statistically significant differences in the mean response between factors were fixed at a $5.0 \%$ significance level threshold $(\mathrm{p}$ value $<0.05)$. The method used to discriminate between the means at the $95.0 \%$ confidence level was Fisher's least significant difference (LSD) procedure. Statistical data analyses were performed using the Statgraphics Centurion XVII (version 17.2.04) statistical software (2014, Statpoint Technologies, Inc., Warrenton, VA).

\section{Results and discussion}

\subsection{Short-term BSA adhesion test}

The effect of different coatings and materials on short-term BSA adhesion was evaluated as described in Section 2.3. The maximum adhesion $\left(0.03 \mu \mathrm{g} \mathrm{mm} \mathrm{mm}^{-2}\right)$ was measured in $\mathrm{HX}(p<0.05$, one-way ANOVA). The BSA adhesion values relative to HX are shown in Figure 1. The minimum adhesion was determined for $\mathrm{H}-\mathrm{X} 3$, being one order of magnitude lower than that in the HX sample $(p<0.05$, one-way ANOVA) (Fig. 1A). There were no statistically significant differences between PMMA, PETG and GS $(p<0.05$, one-way ANOVA).

Protein adsorption on surfaces is the primary event in biofouling. Consequently, low protein adsorption should be recognized as the most important prerequisite for a surface's resistance to biofouling. As PBRgrown microalgae are known to secrete proteins to the supernatant (Xiao and Zheng, 2016), protein adhesion is an expected process. The importance of this phenomenon in microalgae cultures was revealed in a recent study on $N$. gaditana adhesion dynamics (Zeriouh et al., 2017a). The surface of $N$. gaditana was clearly hydrophilic throughout the culture. However, the substrate surface (glass), which was initially hydrophilic, turned almost amphiphilic after two days of culture and strongly hydrophobic a week later. This behavior could only be compatible with the additional adhesion of other materials with hydrophobic properties such as proteins, most likely excreted by $N$. gaditana .

Consequently, PBR surfaces should be designed or selected to resist protein adsorption. However, the control of this multifactorial process is so complex that there is no known surface with complete protein resistance. The hydrophobicity and hydrophilicity of a surface, generally referred to as wettability, are important factors affecting protein adsorption (Firkowska-Boden et al., 2018). The static water contact angle $\vartheta_{\omega}$ is commonly 
used as a descriptor of wettability. Figure $1 \mathrm{~B}$ shows the relative BSA adhesion versus $\vartheta_{\omega}$ for each surface used in this study. A cutoff of $\vartheta_{\omega}$ [?] $65^{\circ}$ was inserted to distinguish between hydrophilic $\left(\vartheta_{\omega}<65^{\circ}\right)$ and hydrophobic $\left(\vartheta_{\omega}>65^{\circ}\right)$ surfaces, as previously reported (Vogler, 2012). The data did not present a clear pattern. The highest BSA adhesions were observed in the most hydrophobic surfaces (HX and NW). This is consistent with the literature since it is expected that proteins undergo partial unfolding and spreading on strongly hydrophobic surfaces (Firkowska-Boden et al., 2018). In contrast, the glass surface (GS), which is strongly hydrophilic, did not show a marked efficacy in reducing BSA attachment compared to the other surfaces. It is supposed that hydrophilic surfaces promote easier protein desorption because the proteins are shielded from the surface by a high-density water layer (Firkowska-Boden et al., 2018). Therefore, GS and PMMA failed as hydrophilic surfaces to inhibit or mitigate the protein adhesion. Surprisingly, BSA adhesion on PS, a moderately hydrophobic solid material, was lower than the hydrophilic surfaces (GS and PMMA). This odd result has so far remained a controversial issue in the field of protein adsorption. Recent studies have revealed that the extent of protein adsorption can be governed by the synergistic effect of the surface hydrophobicity and the relative charge state of the protein and surface (Attwood et al., 2019); so, just because a surface is hydrophilic does not mean it is protein resistant. In fact, globular protein adsorption onto hydrophobic solid surfaces occurs regardless of the protein charge state. Conversely, the adsorption charge of hydrophilic surfaces might be very high under charge-favorable conditions, or very low under unfavorable charge conditions (Attwood et al., 2019). Notwithstanding, in our study, the adhesion propensity of BSA molecules to both hydrophobic and hydrophilic surfaces was significant, the interaction being much stronger on the hydrophobic surfaces. Indeed, this property is exploited in immunology where BSA is used as a blocking agent on both hydrophobic and hydrophilic surfaces (Jeyachandran et al., 2009). Additionally, phosphate groups can be bound with the BSA molecules to form BSA-phosphate surface complexes, the preferred conformation of adsorbed BSA on surfaces (Jeyachandran et al., 2009). Phosphate is the P-source used in the $N$. gaditanaculture medium.

Interestingly, H-X3 (which is hydrophobic) had the lowest BSA adherence propensity. H-X3 is a fouling release coating based on silicone hydrogel. According to recent studies, comparing the protein adsorption results of hydrogels and other non-hydrogel materials, based on a scale of hydrophobicity and hydrophilicity, could be elusive because hydrogels absorb water and create a deformable surface (i.e. water-swollen coatings) (Vogler, 2012).

Another way to quantitatively represent the surface-thermodynamic hydrophobicity/hydrophilicity scale of condensed-phase materials is the interfacial free energy of interaction between two surfaces, i, immersed in water, $\Delta \mathrm{G}_{\mathrm{iwi}}$ (commonly referred to as the free energy of cohesion) (Van Oss, 2008). Figure 1C shows the relative BSA adhesion values as a function of the corresponding $\Delta \mathrm{G}_{\mathrm{iwi}}$ values for each assayed surface. Although the distribution of the experimental points was different to that of $\vartheta_{\omega}$ (Fig. 1B), the conclusions are similar. Only GS presented a clearly positive $\Delta \mathrm{G}_{\mathrm{iwi}}$ value $\left(\Delta \mathrm{G}_{\mathrm{iwi}}>0\right)$, indicating that it is strongly hydrophilic. PMMA presented an $\Delta \mathrm{G}_{\mathrm{iwi}}$ value close to 0 , but as its $\gamma_{s}^{-}$value $\left(27.7 \mathrm{~mJ} \mathrm{~m}^{-2}\right)$ was not significantly greater than the $30 \mathrm{~mJ} \mathrm{~m}{ }^{-2}$ cut-off, it is most likely hydrophobic, as suggested elsewhere (Van Oss, 2008).

From Figures 1B and 1C, one can conclude that criteria based on hydrophobicity and hydrophilicity are not enough to completely explain the protein adhesion on the surfaces. Protein adsorption behavior is a poorly understood and very complex process that can be influenced by diverse factors, acting either synergistically or antagonistically, such as protein molecular properties, surface chemistry and charge, wettability, and topography (Firkowska-Boden et al., 2018). For example, polymers exhibiting similar surface hydrophobicity and roughness, differing only in the chemical end groups, can have divergent protein adsorption profiles (Vijaya Bhaskar et al., 2015). What is even more disconcerting is observing how the same polymer provided by different commercial suppliers can present very different protein adhesion values (Vijaya Bhaskar et al., 2015), likely attributed to minor differences in one or more of the above-mentioned factors, or to a variety of chemical residues generated during manufacturing, which cause the protein to interact differently with the groups on the surface (Contreras-Naranjo and Aguilar, 2019). Interestingly, it is well-known that protein adsorption increases along with the water's ionic strength. Studies have reported that BSA adsorption on 
glass was markedly higher in seawater than in low ionic-strength buffer (Kirchman et al., 1989). The most common explanation is based on the double layer theory: the surface charge of BSA increases in seawater (i.e. a higher ionic strength), causing a reduced repulsive double-layer interaction and a more globular BSA configuration (Kirchman et al., 1989). The results from Figure 1B are consistent with others obtained at ionic strengths as high as seawater (Ruckenstein and Berim, 2019): the greatest BSA adsorption took place on the hydrophobic surfaces, was substantial on glass and significantly lower on the hydrogels. Recent observations support the hypothesis that BSA in seawater forms a multilayer structure involving $\mathrm{Mg}^{2+}$ cations as bridges between the BSA molecules (Pradier et al., 2002). Furthermore, it has been demonstrated that the salts dissolved in seawater produce micrometer-size surface spots containing mainly metal species, these spots being preferential adsorption sites for BSA (Poleunis et al., 2002).

Figure 2 summarizes in an idealized way how the interaction of both the protein and the surface properties may tune the adsorption process, as conceptualized elsewhere (Contreras-Naranjo and Aguilar, 2019). One can observe how the proteins' versatile nature is related to their primary structure, ultimately represented by the sequence of amino acids and functional groups available for bonding. This hinders protein adsorption prevention and leads to experimental data spreading, generated from different laboratories studying the same protein-surface system but from different manufacturers.

\subsection{Long-term biofouling tests}

\subsubsection{Culture experiments in the vessels}

The biofouling tests consisted of exposing the coupons to the $N$. gaditana cultures for a long period (50 days), as explained in Section 2.4. Characterizing these cultures is essential for analyzing the adhesion results based on the interaction between the coupon surface and the broth that surrounds it. The representative culture kinetics for all the vessels are displayed in Figure 3. The experiments started as batch cultures (Stage S1) to acclimate the cells to the new set conditions in the vessels. After 18 days, the fed-batch mode was established. Two nutrient pulses were performed - on days 18 (Stage S2) and 33 (Stage S3). Given that the differences between the experiments that had an initial N/P from 45 to 90 were not significant compared to those with an N/P of 5 and 15, Figure 3 shows the mean values together of the three N/P 45 to 90 . The highest growth rates and biomass concentrations were obtained at an N/P[?]15 in all of the S1 to S3 stages (Fig. 3A).

As can be seen in Figure 3B, the dissolved phosphate was rapidly taken up soon after being added to the cultures with $\mathrm{N} / \mathrm{P}>15$ compared to $\mathrm{N} / \mathrm{P}=5$. At the end of each stage, the phosphate was almost completely consumed at $\mathrm{N} / \mathrm{P}$ values above 5 (Fig. $3 \mathrm{~B}$ ) whereas the dissolved nitrate continued to be present in excess in all experiments (Fig. 3C). The culture dynamics presented above describe a growth pattern that is clearly controlled by the phosphate availability in the culture medium for the experiments with an N/P $>15$. Conversely, growth in the culture medium with an N/P of 5 seemed to be inhibited by phosphate. The $F_{v} / F_{m}$ did not change significantly throughout the culture period, the average value of all vessels being $0.549+-0.035$, which is indicative of healthy cells; the one exception was the value of 0.4 with N/P 5 during S1 and S2.

The coupons were detached from the vessels at the end of stage S3 and the cell adhesion intensity was analyzed as a proxy for microalgal biofouling, as indicated in Section 2.4.2. The factors that could potentially affect the fluid dynamics of biofouling formation on the coupon surface are: the coupon position in the culture vessel (bottom or wall), the coupon surface properties represented by the coupon type, and the culture properties to which the coupons are exposed, represented by the $\mathrm{N} / \mathrm{P}$ ratio. Therefore, to analyze the effect of these factors, and their interactions on the variability of the cell adhesion intensity (AI), a multifactor ANOVA was carried out. All factors and interactions had a statistically significant effect on AI at the $95.0 \%$ confidence level $(\mathrm{p}<0.05)$. However, the most significant contributions to AI variability corresponded to the N/P (27.31\%), the coupon type (23.78\%) and the N/P interaction with the coupon position (12.71\%). The $\mathrm{N} / \mathrm{P}$ factor is a vehicle to combine into one variable the different alterations affecting the environment that directly interacts with the coupons (cells and supernatant) due to the effect of $\mathrm{N}$ on cell metabolism. Nutrient availability, biomass concentration and EOS concentration in the culture were taken into account 
as cellular responses. Figure 4 shows the effect of the above-mentioned factors on AI, EOS, and cell biomass concentrations at the end of the culture experiments.

The cell adhesion intensity and the secreted protein concentration varied inversely to N/P (see Fig. 4A), with no significant effect from N/P ratios above $15(p<0.05)$, while an opposite effect on the final biomass concentration was observed. This implies that cell adhesion correlated positively with the concentration of proteins, but negatively with the concentration of biomass in the culture. The latter seems to contradict previous observations, where it was suggested that an increased cell flow density reaching a coupon surface, governed by cell concentration gradients, increased the cell adhesion rate (Zeriouh et al., 2019b). These seemingly contradictory results are easily reconciled if the protein adhesion that occurred during the formation of the conditioning film preceded cell adhesion.

Considering the results observed in the short-term BSA adhesion tests, the above assumption is highly plausible. A recent study supports this point, in which proteins secreted by the cyanobacterium Annabaenasp were first adsorbed onto suitable PBR materials during the formation of the conditioning film (Talluri et al., 2020). Figure 4A demonstrates that protein adsorption on the coupon surface increased as protein concentration increased in the supernatant, as reported for BSA on surfaces immersed in seawater (Kirchman et al., 1989).

The stickiness of proteins on the different surfaces tested was previously evidenced in Section 3.1, even on hydrophilic surfaces. Therefore, it is expected that $N$. gaditana will adhere to the coupons in proportion to the amount of proteins adhered to them, even on hydrophilic surface (Zeriouh et al., 2017a). This means that $N$. gaditana attachment on the coupon surface is mediated by prior protein adsorption, which occurs at a rate faster than $N$. gaditanaadhesion; that is to say, cells grown in media with N/P ratios of 5 and 15 interacted firstly with the coupon surfaces coated with a conditioning film composed of sticky proteins. In this scenario, the importance of the flow densities of nutrients reaching the cells that were attached to the coupon surface was greater in cultures with the lowest N/P ratios, where nitrates and phosphates were in excess (see Fig. $2 \mathrm{~B}$ ), while in cultures with high $\mathrm{N} / \mathrm{P}$ ratios (above 15), phosphate was present in the supernatant at very low concentrations. As a result, in cultures with an N/P above 15, the microalgae adhering to the coupon surfaces (or embedded in the formed biofilm) continuously experienced significantly reduced phosphate flux densities, which limited their growth. The role played by the excess phosphate present in cultures with an $\mathrm{N} / \mathrm{P}$ below 15 may also have been relevant during protein adsorption if the proteins secreted by N. gaditana had the capacity to bind with the phosphate groups to form protein-phosphate, as mentioned above for BSA. These explanations are consistent with Figure 4B, where the effect of the coupon surface properties (i.e. the type of coupon) on the cell adhesion intensity is observed. The resulting pattern resembled that of BSA adsorption on the same type of coupon represented in Figure 1A.

The effect of the coupon position on cell adhesion intensity is shown in Figure 4C. One can observe that the slight variability in the cell adhesion intensity associated with the coupons' position (bottom or wall) mainly occurred in the experiments with an NP $=5$, where the highest EOS excretion took place.

It has been suggested that wall shear influences cell attachment to the bottom well plates when agitated on a shaking table (Saleck et al., 2011). The strain rate values were always higher on the coupons attached to the flask wall than those on the bottom (Table 2).

All the wall values were almost twice those on the bottom. The differences were higher for the maximum ( 2.7 times) and minimum ( 6.2 times) values. It has been posited that the initial adhesion might be reversible if the kinetic energy of the microalgae cells in the boundary layer of the solid were superior to the total interaction energy between the solid surface and the microalgae cell. The strain rate $(\gamma) 5 \mu \mathrm{m}$ from the coupons is indicative of the drag force exerted by the liquid flow on the boundary layer of the solid surface (Zeriouh et al., 2017b). However, it has been suggested that the shear stress factor alone is not sufficient to explain what was observed occurring in the different attachment experiments. Nonetheless, when linked to flow field topology, it helps in understanding the underlying mechanisms of the different biological processes (Salek et al., 2011). Hence, it is necessary to consider both the strain rate values and the different flow 
structures developed on the bottom and walls of the vessel (Chakraborty et al., 2011). Usually, in orbitally agitated devices, the liquid height is low and a thin liquid film periodically sweeps the bottom, provoking the formation of a radial shear gradient (Salek et al., 2011). The trailing wave formed at the liquid surface forms a recirculation cell that increases the interaction of the cells with the vertical wall of the vessel (Salek et al., 2011). The liquid wetting the coupons on the walls and the bottom of the vessel does not have the flow influence associated with the liquid-gas interphase (Fig. 5B); therefore. the liquid velocity at the bottom is practically constant across the entire cross section (Fig. 5C). The coupons on the vertical wall, however, are closer to the liquid free surface and are subjected to a periodic velocity radial gradient, which generates higher strain rates (Fig. 5D and Table 2), very similar to that occurring in shallow agitated wells (Salek et al., 2011, Berson et al., 2008). In addition, the trailing wave of the liquid surface generates a recirculation cell that repeatedly throws the cells against the wall (Salek et al., 2011, Kim and Kizito, 2009), to some degree centrifuging the cells onto their surface (Kim and Kizito, 2009), which would promote cell adhesion. The different liquid flows developed in the different parts of the flasks apparently counteracted the different strain rate values and, as a consequence, cell attachment was similar on all the coupons.

When the protein concentration in the culture is very high $(\mathrm{N} / \mathrm{P}=5)$ (Fig. $4 \mathrm{~A})$, the fluid flow also seems to carry more proteins towards the wall coupons; therefore, interaction with the cells is higher, as is the cell attachment, compared to the coupons on the bottom (Fig. 4C).

\subsubsection{Nitrogen balance}

The synthesis of intracellular components and the release of extracellular organic substances is mediated by nitrogen availability to the cells. As the nitrogen added to a culture is not only fixed in proteins, the nitrogen balance needs to be acceptably closed. The nitrogen taken up by the cells can be distributed amongst the main extracellular and intracellular proteinaceous and non-proteinaceous nitrogenous compounds, namely proteins, amino acids, chlorophylls, nitrate, nitrite and ammonium.

The analyses carried out to determine the total nitrogen present in both the biomass and the supernatant have revealed that, in general, this element has been taken up in a similar way for all the N/P ratios: 10-13\% has been used to form part of the biomass (both cells in suspension and those attached to the vessel), while $87-90 \%$ was used in the extracellular medium. Furthermore, one should point out that only 25-35\% of the nitrogen was released into the medium as nitrate (not consumed), nitrite and ammonium; that is to say, the rest of the nitrogen (65-75\%) went to forming part of the organic compounds (proteins, ammonia acids, chlorophylls, DNA fragments and lipids, etc.).

As for organic nitrogen, this element has also been distributed similarly for all the N/P ratios. The most appreciable difference is that at the $\mathrm{N} / \mathrm{P} 5$ ratio, $50 \%$ of the intracellular nitrogen is present in the adhered cells; this is because there are twice as many adhered cells as there are in suspension. This can be explained by the fact that in this culture, there was $10 \%$ more extracellular protein than in the other cultures and, as previously mentioned, this substance has a great influence on cell adhesion.

\subsubsection{Analysis in the context of the Baier and Vogler biocompatibility theories}

A work recently carried out on $N$. gaditana has demonstrated that the surface biocompatibility theories of Baier and Vogler may offer interesting insights into developing antifouling surfaces for microalgae photobioreactors (Zeriouh et al., 2019a). Figure 6 shows the relative adhesion values as a function of the water adhesion tension (Vogler's theory) and the critical surface tension (Baier's theory). The microalgae adhesion was calculated based on the maximum obtained at the minimum N/P ratio of $5\left(4.06 \cdot 10^{5}\right.$ cells $\left.\mathrm{cm}^{2}\right)$. For clarity's sake, only the results corresponding to the $\mathrm{N} / \mathrm{P}$ ratios that led to the highest $(\mathrm{N} / \mathrm{P}=5)$ and the lowest $(\mathrm{N} / \mathrm{P}=90)$ cell adhesion and secreted protein concentration values were represented. For the BSA protein model, the values represented in Figure 1 were used. One can observe that the Baier and Vogler theories are only reconciled at the Baier curve minimum attained by the H-X3 coating, based on FRCs-Hydrogel. The exceptional antibiofouling properties of H-X3 have recently been demonstrated in N. gaditanacultures (Zeriouh et al., 2019a). H-X3, although initially having low energy surface properties $\left(\gamma_{S}=22 \mathrm{~mJ} \mathrm{~m}^{-2}\right.$ and $\tau_{0}=-21.2 \mathrm{~mJ} \mathrm{~m}^{-2}$ ), can acquire amphiphilic properties after exposure to seawater; these properties are close 
to Vogler's minimum ( $\tau_{0}$ of around $35 \mathrm{~mJ} \mathrm{~m}^{-2}$ ), as reported by Zeriouh et al. (2019a) (see Figure 6). The BSA and cell adhesion intensity on the rest of the coupons in the vicinity of Vogler's minimum were clearly several orders of magnitude higher than that of H-X3. As mentioned above, these analyses, which are based exclusively on surface wettability, are not universal. Even though there is a substantial body of literature dealing with the topic of protein adsorption on surfaces, in a recent excellent review, Vogler confirmed the huge lack of consensus and the impossibility of extracting general conclusions beyond a few general rules of thumb based on the biophysical chemistry of protein adsorption (Vogler, 2012).

Likewise, two further observations from Figure 6 are of particular importance: (i) the pattern of relative BSA adsorption intensity clearly resembles those of the different $\mathrm{N} / \mathrm{P}$ ratios assayed; and (ii) the higher the concentration of proteins in the supernatant (high for 5 and low for 90, see Fig. 4), the higher the cell adhesion.

These observations support the assumption that the initial event that initiates the biological response of an $N$. gaditana culture to artificial materials and coatings is the adsorption of proteins secreted by the microalgae into the culture medium. This is in accordance with results from studies on photobioreactor materials used in the cultivation of the cyanobacterium Anabaena sp., where proteins were present on the conditioning films formed on glass and PMMA (Talluri et al., 2020).

\subsubsection{Short-term BSA adhesion is indicative of the microalgal biofouling propensity}

Given that protein-resistant materials and coatings are also likely to be resistant to microalgae adhesion, two experimental approaches were addressed in this study: the short-term batch adhesion of the sticky BSA model protein under dynamic conditions and the long-term microalgal adhesion under flow conditions that are representative of practical operation. Relative BSA adhesion tended to approximate the Baier curve and successfully mimic the long-term biofouling test results (Fig. 6). These results demonstrate that the short-term batch adhesion test of a sticky model protein such as BSA is a proxy for predicting biofouling in microalgae cultures. Therefore, long-term representative studies may not be mandatory as a preliminary step in assessing potential biofouling control in strategies applied to the massive screening of surfaces.

\subsection{Future prospects}

The work reported here indicates that, to effectively design or select materials and coatings for use in PBR construction, protein adsorption is a more critical event to consider than that of the cell/clean surface interaction. When PBR surfaces are exposed to cultures containing EOS, including proteins, they are likely to be rapidly coated by a thin layer comprised mainly of proteins dissolved in the supernatant which adsorb to the PBR surface as a monolayer or multilayer. The process of cell adhesion to the surface is indirect because the cells do not bind directly to the PBR surface but instead bind to the adsorbed proteins. According to Vogler (Vogler, 2012), adsorbed protein on water-surface interphases displaced an equivalent volume of interphase water (interphase dehydration), causing adsorbed proteins to concentrate into closely-packed arrangements. Microalgae make contact with, and anchor to, the adsorbed proteins in certain patches and domains.

Although BSA represents an interesting laboratory model for globular protein, the composition, structure, chemistry and functions of the proteins contained in EOS is, as yet, little known (Xiao and Zheng, 2016). Furthermore, the type and profile of the proteins secreted by cultured microalgae may be dependent upon the abiotic conditions and species. Consequently, to gain further insight, studies on the adsorption of other model globular proteins may be needed, as is recommended for animal cells (Attwood et al., 2019). In addition, a conditioning film may also comprise other macromolecules, such as humic substances, polysaccharides, or free amino acids present in the water (Flemming, 2002). All of these can be excreted by the microalgae at high concentrations in PBR cultures. Generally, the adsorption process of these macromolecules onto surfaces (within seconds or minutes) is faster than the cell adhesion (Flemming, 2002). Hence, the degree of initial macromolecule attachment might also be a good indicator of biofouling and its study should therefore be addressed. 
Microalgae respond to changing environmental conditions by modulating their extracellular and intracellular metabolites. Within the same microalgal species, the optimal culture conditions vary depending on the target metabolite. Therefore, besides the $\mathrm{N} / \mathrm{P}$ ratio in the culture medium, the possibilities of combining a wide variety of abiotic factors are vast. Each of these combinations most likely modifies the interaction between the PBR surface and the culture due to changes in the culture and cell properties. From a practical point of view, the biofouling propensity of different PBR surfaces should be studied specifically for each cultivation environment and species in order to the design and/or select the most appropriate surfaces or coatings. Likewise, transparent H-X3-like coatings should be developed and tested for PBR use in microalgae cultures where biofouling is likely dominated by EOS.

\section{Conclusions}

Relative BSA adhesion and microalgal biofouling intensities resemble the Baier curve. The Baier minimum was achieved with the H-X3 coating based on FRC hydrogel. These results demonstrate that the short-term BSA batch test can serve as a proxy to predict microalgae culture biofouling. Vogler's biocompatibility theory only reconciled Baier's theory for the H-X3 coating. All the rigid materials and coatings failed to completely prevent BSA protein adhesion and microalgal biofouling. Adsorption of extracellular proteins secreted by $N$. gaditana most probably occurred prior to cell adhesion. Fine-tuning the N/P ratio might be an interesting way to modulate sticky protein production (and EOS in general) in N. gaditana cultures. Avoiding early protein adhesion should be a preliminary step to designing or selecting materials and coatings for the manufacture of photobioreactors.

\section{Acknowledgements.}

This work was supported by the Spanish Ministry of Science and Innovation (grants RTI2018-101891-B-100 and PID2019-109476RB-C22 BIOMAGRI), and the European Regional Development Fund Program.

\section{References}

APHA. (1995). Standard Methods for the Examination of Water and Wastewater, 19th ed. APHA, New York.

Attwood, S.J., Kershaw, R., Uddin, S., Bishop, S.M., Welland, M.E., (2019). Understanding how charge and hydrophobicity influence globular protein adsorption to alkanethiol and material surfaces. Journal of Materials Chemistry. B, 7(14), 2349-2361.

Berson R.E., Purcell M.R., Sharp M.K., (2008). Computationally Determined Shear on Cells Grown in Orbiting Culture Dishes, in: Kang K.A., Harrison D.K., Bruley D.F. (Eds), Oxygen Transport to Tissue XXIX. Advances In Experimental Medicine And Biology, vol 614. Springer, Boston, MA.

Camacho-Rodríguez, J., Cerón-García, M., González-López, C., Fernández-Sevilla, J., Contreras-Gómez, A., Molina-Grima, E., (2013). A low-cost culture medium for the production of Nannochloropsis gaditana biomass optimized for aquaculture. Bioresource Technology , 144, 57-66.

Chakraborty, A., Chakraborty, S., Jala,V.R., Haribabu, B., Sharp, M.K., Berson, R.E., (2012). Effects of biaxial oscillatory shear stress on endothelial cell proliferation and morphology. Biotechnology and Bioengineering , 109(3):695-707.

Contreras-Naranjo, J.E., Aguilar, O., (2019). Suppressing non-specific binding of proteins onto electrode surfaces in the development of electrochemical immunosensors. Biosensors (Basel), 9(1), 15.

D1426-08, A. (2008). Standard Test Methods for Ammonia Nitroen In Water, ASTM International, West Conshohocken, PA, www.astm.org.

Eilers, P.H.C., Peeters, J.C.H., (1988). A model for the relationship between light intensity and the rate of photosynthesis in phytoplankton. Ecological Modelling , 42(3-4), 199-215. 
Firkowska-Boden, I., Zhang, X., Jandt, K.D., (2018). Controlling protein adsorption through nanostructured polymeric surfaces. Advanced Healthcare Materials, 7(1), 1700995.

Jeyachandran, Y., Mielczarski, E., Rai, B., Mielczarski, J., (2009). Quantitative and qualitative evaluation of adsorption/desorption of bovine serum albumin on hydrophilic and hydrophobic surfaces. Langmuir , 25(19), 11614-11620.

Kim, H.M. \& Kizito, J.P., (2009). Stirring free surface flows due to horizontal circulatory oscillation of partially filled container. Chemical Engineering Communication , 196(11), 1300-1321.

Kirchman, D.L., Henry, D.L., Dexter, S.C., (1989). Adsorption of proteins to surfaces in seawater.Marine Chemistry , 27(3-4), 201-217.

Lopez-Rosales, L., Gallardo-Rodriguez, J.J., Sanchez-Miron, A., Ceron-Garcia, M.C., Belarbi, E.H., GarciaCamacho, F., Molina-Grima, E., (2014). Simultaneous effect of temperature and irradiance on growth and okadaic acid production from the marine dinoflagellate Prorocentrum belizeanum. Toxins, 6(1), 229-253.

Molina-Miras, A., Lopez-Rosales, L., Sanchez-Miron, A., Ceron-Garcia, M.C., Seoane-Parra, S., GarciaCamacho, F., Molina-Grima, E., (2018). Long-term culture of the marine dinoflagellate microalga Amphidinium carterae in an indoor LED-lighted raceway photobioreactor: Production of carotenoids and fatty acids.Bioresource Technology , 265, 257-267.

Nielsen, P.M., Petersen, D., Dambmann, C., (2001). Improved method for determining food protein degree of hydrolysis. Journal of Food Science, 66, 642-646.

Poleunis, C., Rubio, C., Compere, C., Bertrand, P., (2002). Role of salts on the BSA adsorption on stainless steel in aqueous solutions. II. ToF-SIMS spectral and chemical mapping study. Surface and Interface Analysis , 34(1), 55-58.

Pradier, C., Costa, D., Rubio, C., Compere, C., Marcus, P., (2002). Role of salts on BSA adsorption on stainless steel in aqueous solutions. I. FT-IRRAS and XPS characterization. Surface and Interface Analysis, $34(1), 50-54$.

Ruiz-Cabello, F.J.M., Rodriguez-Valverde, M.A., Cabrerizo-Vilchez, M.A., (2011). Contact angle hysteresis on polymer surfaces: An experimental study. Journal of Adhesion Science and Technology , 25(16), 20392049 .

Ruckenstein, E., \& Berim, G. (2019). Wetting: Theory and Experiments, Two-Volume Set. CRC Press.

Salek, M.M., Sattari, P., Martinuzzi, R.J., (2012). Analysis of fluid flow and wall shear stress patterns inside partially filled agitated culture well plates. Annals of Biomedical Engineering , 40(3), 707-728.

Talluri, S.N., Winter, R.M., Salem, D.R., (2020). Conditioning film formation and its influence on the initial adhesion and biofilm formation by a cyanobacterium on photobioreactor materials. Biofouling, 36(2), 183-199.

Van Oss, C.J. (2008). The properties of water and their role in colloidal and biological systems . Academic Press.

Vijaya Bhaskar, T.B., Roch, T., Romero, O., Ma, N., Kratz, K., Lendlein, A., (2015). Single and competitive protein adsorption on polymeric surfaces. Polymers for Advances Technologies, 26(12), 1387-1393.

Vogler, E.A., (2012). Protein adsorption in three dimensions. Biomaterials, 33(5), 1201-1237.

Wang, D., Wu, X., Long, L., Yuan, X., Zhang, Q., Xue, S., Wen, S., Yan, C., Wang, J., Cong, W., (2017). Improved antifouling properties of photobioreactors by surface grafted sulfobetaine polymers. Biofouling, 33(10), 970-979. 
Wang, Y., Chen, C., Wu, X., Wang, Z., Wen, S., Yu, J., Yan, C., Cong, W., (2020). Improved antibiofouling properties of photobioreactor with amphiphilic sulfobetaine copolymer coatings. Progress in Organic Coatings , 144, 105666.

Xiao, R., Zheng, Y., (2016). Overview of microalgal extracellular polymeric substances (EPS) and their applications. Biotechnology Advances, 34(7), 1225-1244.

Zeriouh, O., Marco-Rocamora, A., Reinoso-Moreno, J.V., Lopez-Rosales, L., Garcia-Camacho, F., MolinaGrima, E. (2019a). New insights into developing antibiofouling surfaces for industrial photobioreactors. Biotechnology and Bioengineering, 116(9) ,2212-2222.

Zeriouh, O., Reinoso-Moreno, J., Lopez-Rosales, L., Sierra-Martin, B., Ceron-Garcia, M., Sanchez-Miron, A., Fernandez-Barbero, A., Garcia-Camacho, F., Molina-Grima, E., (2017a). A methodological study of adhesion dynamics in a batch culture of the marine microalga Nannochloropsis gaditana. Algal Research, 23, 240-254.

Zeriouh, O., Reinoso-Moreno, J.V., Lopez-Rosales, L., Ceron-Garcia, M.C., Sanchez Miron, A., GarciaCamacho, F., Molina-Grima, E., (2019b). Assessment of a photobioreactor-coupled modified Robbins device to compare the adhesion of Nannochloropsis gaditana on different materials. Algal Research, 37, 277-287.

Zeriouh, O., Reinoso-Moreno, J.V., Lopez-Rosales, L., Ceron-Garcia, M.C., Sanchez-Miron, A., GarciaCamacho, F., Molina-Grima, E., (2017b). Biofouling in photobioreactors for marine microalgae. Critical Reviews in Biotechnology, 37(8), 1006-1023.

Zhu, X., Jańczewski, D., Lee, S.S.C., Teo, S.L.M., Vancso, G.J., (2013). Cross-linked polyelectrolyte multilayers for marine antifouling applications. ACS Applied Materials \& Interfaces , 5(13), 5961-5968.

\section{Figure Captions}

Figure 1. (A) Relative adsorption of bovine serum albumin (BSA) on different adsorbent surfaces as (B) a function of the static water contact angle $\left(\vartheta_{\omega}\right)$ and $(\mathbf{C})$ the free energy of cohesion $\left(\Delta \mathrm{G}_{\mathrm{iwi}}\right)$.

Figure 2. Idealized representation of the versatility of the Protein-Surface Interactions (adapted from (Contreras-Naranjo and Aguilar, 2019)).

Figure 3. (A) Freely suspended cell concentration of $N$. gaditana (g/L) during the culture period for the different N/P ratios. (B) Phosphate concentration $\left[\mathrm{PO}_{4}{ }^{-}\right](\mu \mathrm{M})$ during the culture period for the different N/P ratios. (C) Nitrate concentration $\left[\mathrm{NO}_{3}{ }^{-}\right](\mathrm{mM})$ during the culture period for the different N/P ratios. Data points are averaged values, and vertical bars are the standard deviation for the two independent experiments and triplicate samples.

Figure 4. (A) Average cell adhesion (cells $/ \mathrm{mm}^{2}$ ) between all surfaces, biomass concentration (g/L) and protein concentration $(\mathrm{g} / \mathrm{L})$ of the supernatant after 50 days. (B) the influence of the N/P ratio and type of material on cell adhesion (cells $/ \mathrm{mm}^{2}$ ), obtained with a multifactorial ANOVA (C) Average cell adhesion (cells $/ \mathrm{mm}$ ) according to the position for each N/P ratio and multifactorial ANOVA result of position influence on the cell adhesion. The lowercase and capital letters in Figure 5B represent significant differences, with a p-value $<0.05$.

Figure 5. (A) Details of the vessel geometry and distribution of the coupons used for material testing. (B) Detail of the liquid-gas interface once the flow was developed. (C) Velocity vectors for a horizontal plane $5 \mu \mathrm{m}$ above the coupons on the bottom and(D) Velocity vectors for a horizontal plane that passes through the middle of the coupons on the wall.

Figure 6. Relative cell adhesion (\%) for each surface (NW, HX, H-X3, PS, PETG, PMMA and GS) and $\mathrm{N} / \mathrm{P}$ ratio after 50 days, and the relative BSA adhesion (\%) for each surface after $4 \mathrm{~h}$.

\section{Hosted file}


Figure 1.docx available at https://authorea.com/users/354221/articles/477857-long-termbiof ouling-formation-mediated-by-extracellular-proteins-in-nannochloropsis-gaditanamicroalga-cultures-at-different-medium-n-p-ratios

\section{Hosted file}

Figure 2.docx available at https://authorea.com/users/354221/articles/477857-long-termbiofouling-formation-mediated-by-extracellular-proteins-in-nannochloropsis-gaditanamicroalga-cultures-at-different-medium-n-p-ratios

\section{Hosted file}

Figure 3.docx available at https://authorea.com/users/354221/articles/477857-long-termbiofouling-formation-mediated-by-extracellular-proteins-in-nannochloropsis-gaditanamicroalga-cultures-at-different-medium-n-p-ratios

\section{Hosted file}

Figure 4.docx available at https://authorea.com/users/354221/articles/477857-long-termbiofouling-formation-mediated-by-extracellular-proteins-in-nannochloropsis-gaditanamicroalga-cultures-at-different-medium-n-p-ratios

\section{Hosted file}

Figure 5.docx available at https://authorea.com/users/354221/articles/477857-long-termbiofouling-formation-mediated-by-extracellular-proteins-in-nannochloropsis-gaditanamicroalga-cultures-at-different-medium-n-p-ratios

\section{Hosted file}

Figure 6.docx available at https://authorea.com/users/354221/articles/477857-long-termbiofouling-formation-mediated-by-extracellular-proteins-in-nannochloropsis-gaditanamicroalga-cultures-at-different-medium-n-p-ratios

\section{Hosted file}

Table 1.docx available at https://authorea.com/users/354221/articles/477857-long-termbiofouling-formation-mediated-by-extracellular-proteins-in-nannochloropsis-gaditanamicroalga-cultures-at-different-medium-n-p-ratios

\section{Hosted file}

Table 2.docx available at https://authorea.com/users/354221/articles/477857-long-termbiofouling-formation-mediated-by-extracellular-proteins-in-nannochloropsis-gaditanamicroalga-cultures-at-different-medium-n-p-ratios 